\title{
INFLUENCE DU MAINTIEN EN MER DES MÂLES DE SAUMON ATLANTIQUE (SALMO SALAR), PENDANT LA PÉRIODE DE REPRODUCTION, SUR LA QUALITÉ DU SPERME.
}

\author{
G. MAISSE (1), R. BILLARD (2), J. COSSON (3), C. LABBE (1), M. LOIR (1), \\ F. LE GAC (1) et F. FIERVILLE (2)
}

(1) I.N.R.A., Laboratoire de Physiologie des Poissons, IFR 43, Campus de Beaulieu, 35042 Rennes Cedex, France.

(2) M.N.H.N., Laboratoire d'Ichtyologie Générale et Appliquée, 3 rue Cuvier, 75231 Paris Cedex 03, France.

(3) C.N.R.S., Université Pierre et Marie Curie, Station Marine, 06230 Villefranche-sur-Mer, France.

\section{RÉSUMÉ}

Lors d'une étude antérieure, nous avons montré que le maintien en mer des Saumons atlantiques mâles affecte la qualité du sperme. Pour tester l'hypothèse de l'influence néfaste d'une contamination par l'urine, nous avons comparé la qualité du sperme collecté par la technique classique (pression des flancs ou " stripping ") à celle du sperme prélevé directement dans les canaux déférents, ceci chez des géniteurs, les uns gardés en mer et les autres transférés en eau douce un mois plus tôt. Alors que les spermes prélevés dans les canaux déférents des deux lots de mâles (eau douce : ED ; eau de mer : EM) ont un spermatocrite, une motilité, un contenu en ATP et une fécondance (en frais) peu différents, ces quatre paramètres sont significativement plus faibles pour les spermes obtenus par " stripping " des mâles en mer. L'analyse des données permet de conclure que certains de ces spermes ont été contaminés par l'urine et l'hypothèse est proposée que la teneur élevée en magnésium de l'urine a pu entraîner une activation des gamètes et une chute de leur ATP les rendant inféconds. En outre, il est apparu que l'aptitude à la congélation des spermes prélevés dans les canaux déférents est plus faible pour les mâles en mer que pour les mâles en eau douce. Les rapports molaires cholestérol/phospholipides des membranes des gamètes, susceptibles d'intervenir dans la résistance au froid, ont été mesurés. Aucune différence significative n'a été trouvée entre les spermes ED et EM.

\section{INFLUENCE ON THE SEMEN QUALITY OF MAINTAINING MALES OF ATLANTIC SALMON, SALMO SALAR, IN SEA WATER DURING THE BREEDING SEASON.}

\section{ABSTRACT}

We have shown previously that the semen collected from male Atlantic salmon kept in sea water has often a low quality. To check for the hypothesis that this could be due to contamination by urine, we have compared the characteristics of semen collected by 
stripping, with those of semen collected directly in the deferent ducts, from males maintained in sea water or transferred to freshwater one month before. While the semen taken in the ducts of both batches of males (freshwater : ED ; sea water : EM) has similar spermatocrit, motility, ATP content and fertility, these parameters were significantly lower for semen obtained by stripping from males in sea water. The analysis of the data suggests that some of these sperms were contaminated by urine and the hypothesis is proposed that the high magnesium content of urine could have activated the gametes, inducing a low ATP content and a low fertility. In addition, the fertility of frozen sperm from the ducts was significantly lower for the males in sea water as compared to the males in freshwater. The molar ratio cholesterol/phospholipids in the membranes of gametes did not differ for the two batches of semen.

\section{INTRODUCTION}

Pour des raisons pratiques, sanitaires et économiques, les salmoniculteurs souhaitent éviter le retour en eau douce, avant la reproduction, des futurs reproducteurs de Saumon atlantique (Salmo salar). Les premiers travaux que nous avons menés (LE GAC et al., 1992 ; HAFFRAY et al., 1995) ont montré que le maintien en mer des géniteurs affectait significativement la qualité des gamètes et plus particulièrement du sperme de certains mâles, alors que pour d'autres la qualité était identique à celle du sperme de géniteurs ayant été transférés en eau douce au moins un mois avant la période de spermiation. En outre, nous avons montré que des fécondations avec le sperme de Saumons maintenus en mer peuvent être réalisées en utilisant le dilueur DIA 532 du commerce(BILLARD, 1977). Nous avons émis l'hypothèse que les spermatozoïdes de mauvaise qualité n'étaient plus activables au moment des tentatives de fécondation.

Il a été montré que la technique de collecte du sperme par pression des flancs entraîne une contamination par l'urine, même lorsque des précautions sont prises pour tenter de la limiter (RANA, 1995). Afin de tester l'hypothèse de l'influence d'une contamination par l'urine, nous avons mis en place une expérimentation destinée à comparer la qualité du sperme collecté par pression des flancs à celle du sperme prélevé directement dans les canaux déférents, à la fois chez des mâles transférés en eau douce un mois avant l'expérimentation et chez ceux gardés en mer. En outre, nous avons testé l'aptitude à la congélation des spermes prélevés dans les canaux déférents. Le rapport cholestérol/phospholipides des membranes des gamètes, susceptible de moduler cette aptitude (DROKIN et KOPEIKA, 1992), a été déterminé pour ces spermes.

\section{MATÉRIEL ET MÉTHODES}

L'étude a été réalisée en Bretagne au mois de décembre sur des Saumons atlantiques mâles de la souche Norvège et de la souche Elorn, ayant été élevés en mer pendant 18 mois. A cette date, tous les mâles étaient en pleine spermiation. Quinze mâles maintenus en eau de mer, en cages, à la pisciculture de Camaret et dix mâles, qui avaient été transférés en eau douce à la pisciculture fédérale du Favot (Monts d'Arrée) un mois avant les prélèvements, ont été utilisés. Dans les deux structures, les animaux ont été maintenus à jeun pendant le mois précédant l'expérimentation. 


\section{Collecte des spermes}

Après anesthésie dans un bain de $0,03 \%$ de phénoxyéthanol, la papille génitale était séchée et un échantillon de sperme de $10 \mathrm{ml}$ était collecté dans un tube par une pression ménagée des flancs du géniteur (" stripping "). L'animal était ensuite tué par un coup sur la tête et la cavité abdominale ouverte. Dix $\mathrm{ml}$ du sperme présent dans les canaux déférents étaient alors prélevés par aspiration avec une seringue équipée d'une aiguille $(1,2 \times 40)$. Chaque échantillon de sperme "strippé " ou de sperme des canaux déférents était conservé individuellement, sous atmosphère d'oxygène, dans un flacon de culture cellulaire $(50 \mathrm{ml})$, disposé horizontalement sur de la glace fondante.

\section{Spermatocrite}

Un $\mathrm{ml}$ de chaque sperme a été centrifugé $20 \mathrm{~min}$ à $500 \mathrm{~g}$, puis $10 \mathrm{~min}$ à $1000 \mathrm{~g}$. Le “spermatocrite $1000 \mathrm{~g}$ " a été estimé par le rapport du volume du culot (comprenant une forte concentration en spermatozoïdes) au volume total de sperme.

\section{Motilité des spermatozoïdes}

La motilité a été déterminée, par deux observateurs indépendants, par estimation subjective du pourcentage de cellules mobiles et par la durée de la motilité, selon la méthode décrite par BILLARD et COSSON (1992), dans le DIA 532, sur un microscope à contraste de phase (grossissement $\times 160)$. Les observations ont été réalisées à la température de la pièce $\left(18^{\circ} \mathrm{C}\right)$.

\section{Contenu en ATP des spermatozoïdes}

Le contenu en ATP des spermatozoïdes a été estimé selon PERCHEC et al. (1995). Brièvement, un échantillon de sperme est lysé par dilution dans un tampon bouillant (25 mM HEPES, $10 \mathrm{mM}$ acétate de magnésium, $2 \mathrm{mM}$ EDTA, $3 \mathrm{mM} \mathrm{NaN}$, $\mathrm{pH} 7,75$ ). Un $\mu \mathrm{l}$ de luciférine-luciférase (kit de Perstorp S.A.) fraîchement dilué dans le tampon est ajouté à $100 \mu \mathrm{l}$ d'extrait de spermatozoïdes. La luminescence est mesurée dans un Biocounter M2010A Lumac/3M. Le contenu en ATP de chaque sperme est calculé en utilisant un standard interne. Chaque extrait est mesuré en duplicat. La concentration en spermatozoïdes de chaque sperme est déterminée par comptage sur un microscope à contraste de phase à l'aide d'un hématimètre. Les résultats sont exprimés en nmoles d'ATP $/ 10^{8}$ spermatozoïdes.

\section{Fécondance du sperme frais}

La fécondance de chaque sperme a été déterminée à partir du taux d'embryonnement obtenu (moyenne de deux réplicats) par fécondation, à la dilution $10^{-3}$ dans du DIA 532, de 200 ovules provenant du mélange des pontes de plusieurs femelles de la même souche que les mâles. Parallèlement, la fécondabilité des ovules a été évaluée à partir du taux d'embryonnement obtenu par la fécondation de deux lots de 200 ovules avec un mélange de spermes à la dilution $10^{-2}$. Les incubations se sont déroulées dans un circuit fermé thermorégulé à $10^{\circ} \mathrm{C}$. Le taux d'embryonnement a été déterminé par comptage direct des oeufs oeillés après 30 jours. La fécondance de chaque sperme est calculée par rapport à la fécondabilité des ovules. 


\section{Congélation du sperme et fécondance après congélation}

La congélation du sperme a été effectuée rapidement après les prélèvements. Un volume de sperme était dilué avec trois volumes de dilueur de MOUNIB (1978) modifié (LEGENDRE et BILLARD, 1980). La congélation a été réalisée dans des paillettes de $5 \mathrm{ml}$ (IMV, réf. AA303) disposées horizontalement à $3 \mathrm{~cm}$ au-dessus de l'azote liquide pendant $20 \mathrm{~min}$, puis immergées dans l'azote liquide pour $24 \mathrm{~h}$. La fécondance du sperme congelé a été testée en mélangeant $8 \mathrm{~g}$ d'oeufs, $8 \mathrm{ml}$ de DIA 532 à $12^{\circ} \mathrm{C}$ et $320 \mu \mathrm{l}$ du mélange décongelé sperme-dilueur de congélation. Dans ces conditions, la concentration réelle du sperme congelé est de $10^{-2}$, valeur préconisée par LEGENDRE et BILLARD (1980), et le nombre de spermatozoïdes par oeuf était environ $3,2 \times 10^{6}$. Le taux d'embryonnement à 30 jours a été déterminé et la fécondance calculée comme ci-dessus.

\section{Composition lipidique des membranes des spermatozoïdes}

Les membranes des spermatozoïdes ont été préparées par cavitation à l'azote selon LABBE et LOIR (1991). Brièvement, les gamètes sont lavés par centrifugation (15 min à $500 \mathrm{~g}$, puis $5 \mathrm{~min}$ à $1000 \mathrm{~g}$ ) et resuspendus dans une solution de saccharose $0,25 \mathrm{M}$ à pH 7,8 contenant divers inhibiteurs enzymatiques. Trente $\mathrm{ml}$ de la suspension sont soumis à une pression d'azote de 62 bars pendant $20 \mathrm{~min}$. Après décompression rapide, la suspension est homogénéisée doucement dans un potter de Dounce. Quinze $\mathrm{ml}$ de la suspension sont centrifugés comme ci-dessus sur 0,65 volume de saccharose $1 \mathrm{M}$. Le surnageant, enrichi en membranes, est déposé sur un gradient discontinu de saccharose $(0,25-1,8 \mathrm{M})$ et centrifugé $2 \mathrm{~h}$ à $54000 \mathrm{~g}$. Les bandes contenant les membranes sont collectées et les membranes obtenues par centrifugation pendant 20 min à $100000 \mathrm{~g}$. L'analyse des lipides des membranes a été réalisée selon LABBE et LOIR (1991). Les lipides sont extraits dans un mélange chloroforme/méthanol (2v/1v). Les phospholipides sont séparés des triglycérides, des glycolipides et du cholestérol par fractionnement sur cartouche de silice. Le cholestérol total a été dosé par une méthode enzymatique. La quantité de phospholipides a été calculée à partir du phosphate inorganique dosé selon BARTLETT (1959).

\section{Analyse des données}

Pour les séries appariées à l'intérieur de chaque lot de mâles, le test de Wilcoxon a été utilisé. Le test de Mann et Whitney a été utilisé pour la comparaison des données entre lots de mâles.

\section{RÉSULTATS}

Pour les mâles gardés en mer (EM), comme pour les mâles en eau douce (ED), le spermatocrite moyen des spermes obtenus par " stripping" (STR) est significativement inférieur à celui des spermes des canaux déférents (DEF) (Tableau I). Il en est de même en ce qui concerne la motilité dans le cas des mâles EM, celle des gamètes STR étant inférieure à celle des gamètes DEF. Alors que pour les mâles ED, le contenu en ATP des spermatozoïdes ne diffère pas entre les deux méthodes de prélèvement, chez les mâles $E M$, ce contenu est significativement plus faible pour les gamètes STR que pour les gamètes DEF. Pour les deux lots de mâles, la fécondance des spermes DEF est élevée (93 et $95 \%$ ) et ne diffère pas selon l'origine des mâles ; la fécondance des spermes STR leur est significativement inférieure, de $5 \%$ seulement pour les mâles ED contre $32 \%$ pour les mâles EM. 


\section{Tableau I}

Caractéristiques générales des spermes de Saumons atlantiques gardés en mer ou transférés en eau douce un mois avant les prélèvements, et signification statistique des différences observées entre les valeurs moyennes des différents paramètres (test $U$ de Mann et Whitney).

$m=$ moyenne, sem $=$ erreur standard, $v=$ variabilité $(100 \times$ écart type/moyenne $)$, $\mathbf{n}=$ nombre d'individus.

${ }^{\star}: p=5 \% ;{ }^{\star \star}: p=1 \% ;$ NS : non significatif pour $p=5 \%$.

Table I

Characteristics of the milt from Atlantic salmon either kept in sea water or transferred to freshwater one month before sampling, and statistical significance of the differences observed between the mean values of the different parameters (Mann and Whitney $U$ test).

$m=$ mean, sem $=$ standard error of the mean, $v=$ variability $(100 \times$ standard deviation/mean), $\mathbf{n}=$ number.

${ }^{\star}: p=5 \% ;{ }^{\star *}: p=1 \% ;$ NS : not significant for $p=5 \%$.

\begin{tabular}{|c|c|c|c|c|c|c|c|c|c|}
\hline \multirow[b]{2}{*}{ Paramètre } & & \multicolumn{2}{|c|}{ Spermes eau de mer } & \multicolumn{2}{|c|}{ Spermes eau douce } & \multicolumn{4}{|c|}{$\begin{array}{c}\text { Signification } \\
\text { statistique }\end{array}$} \\
\hline & & $\begin{array}{c}\text { “Strippé " } \\
\text { (1) }\end{array}$ & $\begin{array}{l}\text { Canaux } \\
\text { déférents } \\
\text { (2) }\end{array}$ & $\begin{array}{l}\text { "Strippé " } \\
\text { (3) }\end{array}$ & $\begin{array}{c}\text { Canaux } \\
\text { déférents } \\
\text { (4) }\end{array}$ & $1 / 2$ & $3 / 4$ & $1 / 3$ & $2 / 4$ \\
\hline $\begin{array}{l}\text { Spermatocrite } \\
\qquad(\%)\end{array}$ & $\begin{array}{c}m \pm \operatorname{sem} \\
v \\
n \\
\end{array}$ & $\begin{array}{c}25 \pm 2,8 \\
44 \\
15 \\
\end{array}$ & $\begin{array}{c}38 \pm 2,6 \\
26 \\
15 \\
\end{array}$ & $\begin{array}{c}18 \pm 1.7 \\
27 \\
9 \\
\end{array}$ & $\begin{array}{c}23 \pm 1.7 \\
22 \\
9 \\
\end{array}$ & $* *$ & $*$ & NS & $* *$ \\
\hline $\begin{array}{l}\text { Motilité } \\
\qquad \%)\end{array}$ & $\begin{array}{c}m \pm \text { sem } \\
v \\
n \\
\end{array}$ & $\begin{array}{c}32 \pm 8,3 \\
100 \\
15 \\
\end{array}$ & $\begin{array}{c}57 \pm 7,2 \\
49 \\
15 \\
\end{array}$ & $\begin{array}{l}- \\
- \\
-\end{array}$ & $\begin{array}{c}79 \pm 4,2 \\
15 \\
8 \\
\end{array}$ & $* *$ & & & NS \\
\hline $\begin{array}{c}\text { ATP } \\
\left(\mathrm{nmol} / 10^{8} \mathrm{spz}\right)\end{array}$ & $\begin{array}{c}m \pm \text { sem } \\
v \\
n\end{array}$ & $\begin{array}{c}6,0 \pm 1,20 \\
77 \\
15 \\
\end{array}$ & $\begin{array}{c}12,1 \pm 1,05 \\
32 \\
14\end{array}$ & $\begin{array}{c}16,2 \pm 1,88 \\
37 \\
10 \\
\end{array}$ & $\begin{array}{c}15,6 \pm 1,59 \\
32 \\
10\end{array}$ & ** & NS & $*$ & * \\
\hline $\begin{array}{l}\text { Fécondance } \\
\text { sperme frais } \\
(\%)\end{array}$ & $\begin{array}{c}m \pm \text { sem } \\
v \\
n\end{array}$ & $\begin{array}{c}61 \pm 11.1 \\
70 \\
15 \\
\end{array}$ & $\begin{array}{c}93 \pm 1,5 \\
6 \\
15\end{array}$ & $\begin{array}{c}90 \pm 1,7 \\
6 \\
9\end{array}$ & $\begin{array}{c}95 \pm 10,3 \\
1 \\
9\end{array}$ & * & ** & & NS \\
\hline $\begin{array}{l}\text { Fécondance } \\
\text { sp. congelé } \\
(\%)\end{array}$ & $\begin{array}{c}m \pm \operatorname{sem} \\
v \\
n \\
\end{array}$ & & $\begin{array}{c}7 \pm 2,5 \\
100 \\
8 \\
\end{array}$ & & $\begin{array}{c}26 \pm 4,2 \\
42 \\
7 \\
\end{array}$ & & & & $*$ \\
\hline $\begin{array}{l}\text { Cholestérol/ } \\
\text { Phospholipides } \\
\text { (Rap. molaire) }\end{array}$ & $\begin{array}{c}m \pm \operatorname{sem} \\
v \\
n\end{array}$ & & $\begin{array}{c}1,3 \pm 0,20 \\
15 \\
6\end{array}$ & & $\begin{array}{c}1,0 \pm 0,09 \\
22 \\
6\end{array}$ & & & & NS \\
\hline
\end{tabular}



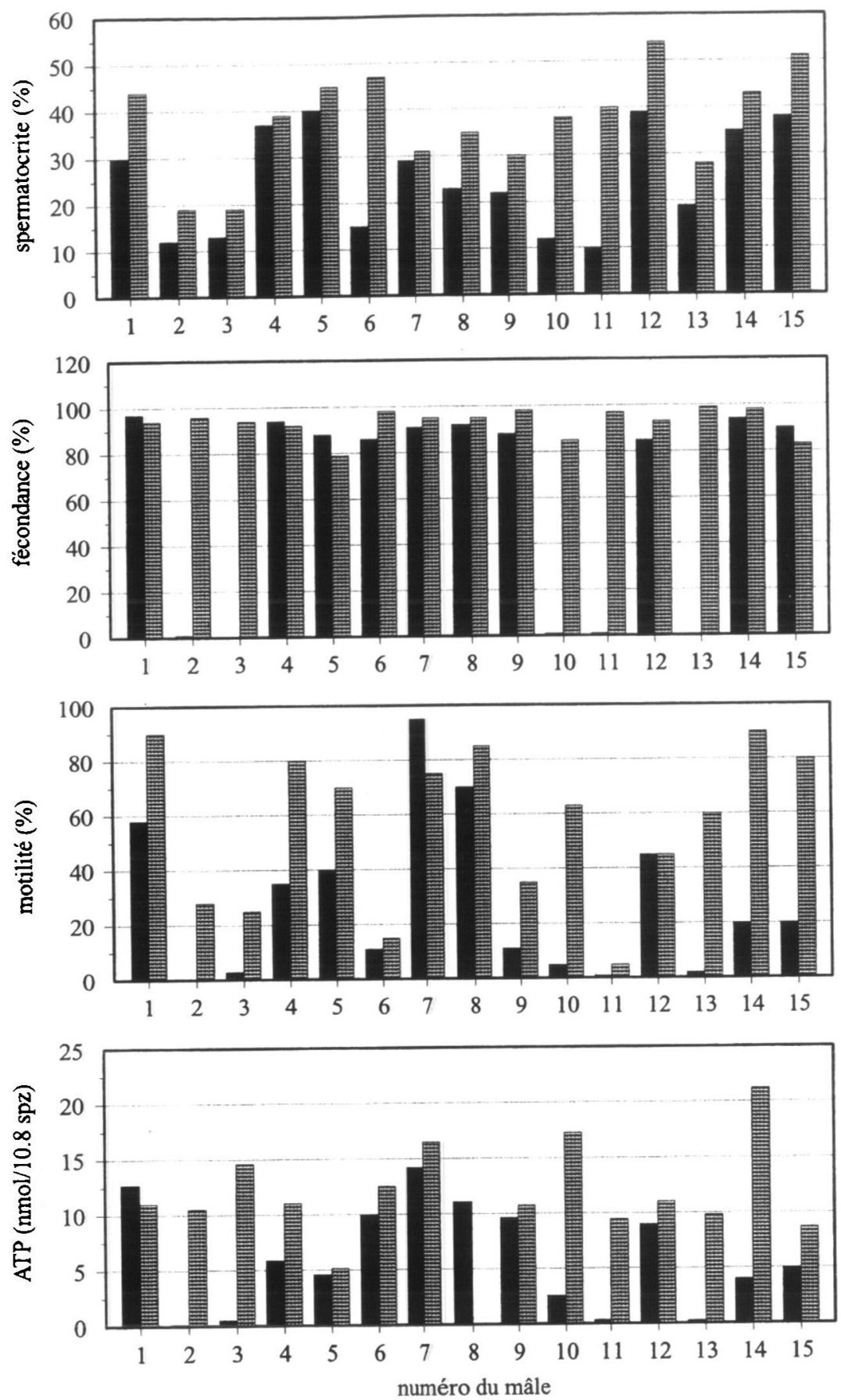

prélevé par «stripping" prélevé dans les déférents

\section{Figure 1}

Spermatocrite, fécondance " en frais ", motilité et contenu en ATP des gamètes des spermes des saumons maintenus en mer (numérotés de 1 à 15). Les spermes ont été collectés soit par pression des flancs (" stripping "), soit directement dans les canaux déférents.

\section{Figure 1}

Spermatocrite, fertility of fresh milt, sperm motility and ATP content of semen from salmon maintained in sea water (numbered from 1 to 15). Semen was collected either by stripping or directly in the deferent ducts. 
Alors que pour les mâles ED, la variabilité (100 $\times$ écart type/moyenne) pour les quatre paramètres étudiés diffère peu entre spermes STR et DEF, ils sont, pour les mâles EM, au moins deux fois plus élevés pour les spermes STR que pour les spermes DEF. Ceci résulte du fait que pour 5 géniteurs $\operatorname{EM}(2,3,10,11$ et 13, Figure 1) sur les 15 testés, les valeurs des paramètres mesurés pour les spermes STR sont très faibles : un spermatocrite moyen de $13 \pm 1,3 \%$, une motilité moyenne de $2,2 \pm 0,85 \%$, un contenu en ATP moyen de $0,8 \pm 0,44 \mathrm{nmole} / 10^{8}$ cellules, et une fécondance moyenne de $0,6 \pm 0,22 \%$. La fécondance moyenne des 10 autres spermes STR-EM est égale à $90 \pm 4 \%$ et ne diffère pas significativement de celle des spermes DEF-EM et STR-ED.

Les spermes DEF de 8 mâles EM et ceux de 7 mâles ED ayant les meilleurs spermatocrites et motilités ont été congelés pour $24 \mathrm{~h}$. Après décongélation, la fécondance des spermes EM a été significativement inférieure à celle des spermes ED (Tableau 1).

Le rapport molaire cholestérol/phospholipides montre une tendance à être supérieur pour les spermes EM que pour les spermes ED, mais la différence n'est pas significative (Tableau 1).

\section{DISCUSSION}

Dans nos conditions de testage du sperme frais, la fécondance du sperme prélevé directement dans les canaux déférents des géniteurs n'est pas différente pour les Saumons atlantiques maintenus en mer ou transférés en eau douce. Pour les animaux en eau douce, le sperme obtenu par "stripping " a un spermatocrite moyen et une fécondance " en frais " moyenne légèrement inférieurs au sperme des canaux déférents, mais les contenus moyens en ATP sont identiques.

Nos résultats confirment la possible médiocrité des spermes collectés par " stripping " chez des Saumons atlantiques mâles maintenus en eau de mer. Cependant, comme nous l'avions déjà observé (HAFFRAY et al., 1995), cette mauvaise qualité ne concerne pas tous les spermes. Dans le cas présent, cinq spermes avaient une fécondance " en frais » nulle alors que celle des dix autres était en moyenne similaire à celle des spermes collectés dans les canaux déférents, ainsi qu'à celle des spermes des mâles en eau douce.

Les cinq spermes STR-EM de mauvaise qualité ont en commun un faible spermatocrite qui suggère qu'ils ont été contaminés par de l'urine pendant le prélèvement, comme il arrive fréquemment lorsque le sperme de Salmonidés est collecté de cette façon (RANA, 1995). La faible motilité de ces spermes est une conséquence de leur très faible contenu en ATP, inférieur à $1 \mathrm{nmole} / 10^{8}$ cellules. II faut noter que chez la Truite arc-enciel, le battement du flagelle stoppe alors qu'il reste pourtant encore $1 / 5^{\mathrm{e}}$ du contenu initial en ATP (CHRISTEN et al., 1987). Nous proposons que cette pauvreté en ATP résulte du fait que les spermatozoïdes ont été activés antérieurement, vraisemblablement lors de la contamination par l'urine. Pour le lot ED, la différence observée au niveau du spermatocrite entre les spermes STR et DEF suggère là aussi une contamination du sperme STR par l'urine dont l'effet semble limité d'après les valeurs proches des fécondances (la motilité n'a malheureusement pas été analysée pour le lot STR-ED). Cependant, à la différence de l'urine des poissons d'eau douce, celle des poissons en mer est caractérisée par une forte teneur en magnésium (110 Mm chez Gillichthys mirabilis, LORETZ et BERN, $1980 ; 120 \mathrm{mM}$ chez Lamprey fluviatilis, RANKIN et al., 1983). Or il a été montré (TANIMOTO et MORISAWA, 1988 ; BOITANO et OMOTO, 1989) que cet ion peut contrer l'inhibition de la motilité des gamètes par le potassium présent dans le fluide séminal. Sur la base de la concentration en magnésium rapportée par LORETZ et BERN, 
un calcul effectué d'après les spermatocrites indique une concentration moyenne en magnésium d'environ $45 \mathrm{mM}$ pour les spermes STR-EM. Cette concentration est supérieure à celle nécessaire pour lever l'inhibition due à $30 \mathrm{mM}$ de potassium (BOITANO et OMOTO, 1989).

A l'opposé de la fécondance " en frais" des spermes DEF, identique pour les deux lots $E M$ et ED, leur aptitude à la congélation est significativement plus faible pour les mâles EM. D'après DROKIN et KOPEIKA (1992), la bonne aptitude à la congélation observée pour le sperme des poissons marins serait due à un rapport molaire cholestérol/phospholipides des membranes plus élevé que celui des poissons d'eau douce. Dans le cas présent, il apparaît 1) que le rapport molaire cholestérol/phospholipides n'est pas significativement plus élevé pour les saumons maintenus en mer que pour les saumons transférés préalablement en eau douce et 2) que bien que ce rapport soit 2 à 3 fois plus élevé chez le Saumon atlantique que chez la Truite (LABBE et LOIR, 1991), les performances des spermes des saumons ED, et a fortiori des saumons EM, sont après congélation-décongélation du même ordre voire inférieures à celles obtenues chez la Truite arc-en-ciel (MALEJAC et al., 1990).

\section{CONCLUSION}

Cette expérimentation confirme le risque d'obtenir des spermes de qualité très médiocre en collectant par "stripping " des géniteurs maintenus en mer. Les solutions consistent, pour éviter les effets néfastes de la contamination par l'urine, à prélever le sperme dans les canaux déférents à l'aide d'un cathéter ou bien à recueillir le sperme " strippé " dans un dilueur de collecte comme le SFMM modifié (OGIER de BAULNY et al., 1997). L'aptitude à la congélation des spermes collectés directement dans les canaux déférents est faible lorsque les géniteurs sont maintenus en mer, par comparaison à ceux transférés en eau douce. Nos données ne permettent pas d'expliquer cette différence qui justifiera des études complémentaires.

\section{REMERCIEMENTS}

Les auteurs remercient pour leur assistance compétente et efficace, le personnel de la Salmoniculture Expérimentale Marine IFREMER-INRA (SEMII).

\section{BIBLIOGRAPHIE}

BARTLETT G.R., 1959. Phosphorus assay in column chromatography. J. Biol. Chem., $234,466-468$.

BILLARD R., 1977. Utilisation d'un système tris-glycocolle pour tamponner le dilueur d'insémination pour truite. Bull. Fr. Piscic., 264, 102-112.

BILLARD R., COSSON M.P., 1992. Some problems related to the assessment of sperm motility in freshwater fish. J. Exp. Zool., 261, 122-131.

BOITANO S., OMOTO C.K., 1989. Ionic requirement for the initiation of trout sperm motility. J. Cell Biol., 107, 168a.

CHRISTEN R., GATTI J.L., BILLARD R., 1987. Trout sperm motility. The transient movement of trout sperm is related to changes in the concentration of ATP following the activation of the flagellar movement. Eur. J. Biochem., 166, 667-671.

DROKIN S.I., KOPEIKA E.F., 1992. Cryoresistance and lipid characteristics of sperm of different fish species. Workshop on gamete and embryo storage and cryopreservation in aquatic organisms, Marly le Roy, 30 mars-4 avril 1992, 50 p. 
HAFFRAY P., FOSTIER A., NORMANT Y., FAURE A., LOIR M., JALABERT B., MAISSE G., LE GAC F., 1995. Influence du maintien en mer ou de la période de transfert en eau douce des reproducteurs de saumon atlantique Salmo salar sur la maturation sexuelle et la qualité des gamètes. Aquat. Living Resour., 8, 135-145.

LABBE C., LOIR M., 1991. Plasma membrane of trout spermatozoa : I. Isolation and partial characterization. Fish Physiol. Biochem., 9, 325-338.

LE GAC F., HAFFRAY P., LOIR M., FOSTIER A., MAISSE G., 1992. Effect of diluent osmolality on the fertilizing ability of spermatozoa in Atlantic salmon Salmo salar kept in sea water or transferred to fresh water. Workshop on gamete and embryo storage and cryopreservation in aquatic organisms, Marly le Roy, 30 mars -4 avril 1992, $5 \mathrm{p}$.

LEGENDRE M., BILLARD R., 1980. Cryoconservation du sperme de truite arc-en-ciel (Salmo gairdneri R.). Bull. Fr. Pisc., 278, 11-33.

LORETZ C.A., BERN H.A., 1980. Ion transport by the urinary bladder of the gobiid teleost, Gillichthys mirabilis. Am. J. Physiol., 239, 415-423.

MALEJAC M.L., LOIR M., MAISSE G., 1990. Qualité de la membrane des spermatozoïdes de truite arc-en-ciel (Oncorhynchus mykiss) ; Relation avec l'aptitude du sperme à la congélation. Aquat. Living Res., 3, 43-54.

MOUNIB M.S., 1978. Cryogenic preservation of fish and mammalian spermatozoa. J. Reprod. Fert., 53, 13-18.

OGIER de BAULNY B., LE VERN Y., KERBOEUF D., MAISSE G., 1997. Flow cytometric evaluation of mitochondrial activity and membrane integrity in fresh and cryopreserved rainbow trout (Oncorhynchus mykiss) spermatozoa. Cryobiology, 34, 141-149.

PERCHEC G., JEULIN C., COSSON J., ANDRE F., BILLARD R., 1995. Relationship between sperm ATP content and motility of carp spermatozoa. J. Cell Sci., 108, 747-753.

RANA K.J., 1995. Preservation of gametes. In BROMAGE N., ROBERTS J. (eds), Broodstock management and egg and larvae quality, 53-75, Blackwell Science, Oxford.

RANKIN J.C., HENDERSON I.W., BROWN J.A., 1983. Osmoregulation and the control of kidney function. In RANKIN J.C., PITCHER T.J., DUGGAN R.T. (eds), Control process in fish physiology, 66-88, Croom Helm, London.

TANIMOTO S., MORISAWA M., 1988. Roles of potassium and calcium channels in the initiation of sperm motility in rainbow trout. Dev. Growth Diff., 30, 117-124. 